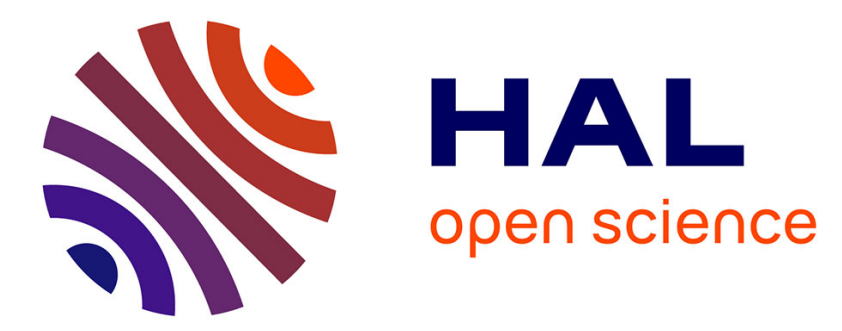

\title{
La schizophrénie à l'écran : processus de subjectivation et de verbalisation dans Clean, Shaven (Kerrigan, 1994) et Spider (Cronenberg, 2001)
}

Jocelyn Dupont

\section{- To cite this version: \\ Jocelyn Dupont. La schizophrénie à l'écran : processus de subjectivation et de verbalisation dans Clean, Shaven (Kerrigan, 1994) et Spider (Cronenberg, 2001). Revue Française d'Etudes Américaines, 2015, 143 (2), pp.72. 10.3917/rfea.143.0072 . hal-02321571}

HAL Id: hal-02321571

https://hal-univ-perp.archives-ouvertes.fr/hal-02321571

Submitted on 2 Jun 2021

HAL is a multi-disciplinary open access archive for the deposit and dissemination of scientific research documents, whether they are published or not. The documents may come from teaching and research institutions in France or abroad, or from public or private research centers.
L'archive ouverte pluridisciplinaire HAL, est destinée au dépôt et à la diffusion de documents scientifiques de niveau recherche, publiés ou non, émanant des établissements d'enseignement et de recherche français ou étrangers, des laboratoires publics ou privés. 


\title{
La schizophrénie à l'écran : processus de subjectivation et de verbalisation dans
}

\author{
Clean, Shaven et Spider
}

\section{Jocelyn Dupont}

\section{Introduction}

Dans le vaste corpus des films qui prennent la psychopathologie comme noyau diégétique et/ou thématique, on peut constater que, quantitativement, la schizophrénie occupe une place de choix. Ceci peut s'expliquer par divers facteurs, à commencer, d'une part, par une certaine coïncidence entre l'émergence de cette catégorie nosologique et celle du cinéma (rappelons que cette pathologie est officiellement intronisée par Bleuler en 1911, faisant suite à la demencia praecox décrite par Kraepelin en 1898) et, d'autre part, en raison du flou qui existe autour de sa définition et continue d’agiter les débats entre spécialistes - faut-il parler de la schizophrénie, des schizophrénies (Marc Louis Bourgeois) ou des « schizophrènes » (Racamier) ? On peut néanmoins gager que la prédilection du cinéma pour cette catégorie psychopathologique particulière s'explique surtout par une assimilation de certains symptômes attachés à la schizophrénie, assimilation le plus souvent réductrice voire stéréotypique, mais néanmoins réelle et féconde.

Parmi ces symptômes les mieux connus, on notera la prévalence des hallucinations, dont le potentiel cinématographique est tout à fait remarquable, les états délusionnels, aptes à générer des récits trompeurs, portés notamment par des narrateurs fous, et surtout la question de la dissociation de personnalité (Spaltung), le clivage schizoïde du sujet, qui, au cinéma, a pu donner lieu à des effets de fragmentation et autres dédoublements spectaculaires, même s'ils constituent souvent des contresens majeurs en termes psychiatriques. Quelques titres comme M. Klein de Joseph Losey, Le Locataire de Roman Polanski, Fight Club de David Fincher, A Beautiful Mind de Ron Howard, ou Black 
Swan de Darren Aronofsky viennent à l'esprit, ainsi que, bien évidemment, Psycho d'Alfred Hitchcock. Son schizophrène assassin Norman Bates fut l'initiateur d'une veine filmique extrêmement prolifique dans laquelle le psychopathe devient tueur sanguinaire - le substantif composé psycho-killer en anglais marque bien la collusion sémantique à l'œuvre - variante contemporaine du monstre et facteur d'inquiétude sociale. Si, selon la thèse de Susan Sontag, le discours de la maladie fonctionne selon un régime métaphorique, alors nous partageons la thèse de Joshua David Bellin quand il écrit que, dans le cas de la maladie mentale, les métaphores de la folie enflent pour devenir (swell into) de purs spectacles de violence ${ }^{1}$. C'est notamment le cas dans le franchement brutal Schizophrenia de l'autrichien Gerald Kargl (1983), récemment ressorti en DVD². De tels films participent pleinement de ce que Sander L. Gilman a justement identifié comme la projection fantasmatique de l'altérité et la construction du stéréotype en opposition au moi afin d'assurer la protection et l'intégrité de ce dernier. Ils illustrent aussi la thèse foucaldienne selon laquelle le fou est associé au criminel dans la construction discursive dominante, et mérite donc d'être enfermé tout en perpétuant des clichés iconographiques et des modes de représentations d'un autre âge, à l'instar de la célèbre gravure de l'anatomiste britannique Charles Bell, réalisée au début du $19^{\text {ème }}$ siècle. Gare au psychokiller près de chez vous.

Bien que quantitativement moins nombreux que les films précédemment cités, certains films choisissent d'explorer la schizophrénie par d'autres voies que celles du stéréotype et de la violence, en mettant plutôt l'accent sur la dimension pathologique de cette affliction mentale mais sans toutefois renoncer à l'élaboration de fictions. C'est le cas, entre autres, de Family Life de Ken Loach ou de Take Shelter de Jeff Nichols, deux films qui incluent le discours sur la maladie mentale dans un cadre social plus vaste. C'est aussi le cas des deux longs métrages qui constituent l'objet principal de cette communication. Les films dont j'ai choisi de parler aujourd'hui poussent d'un cran

\footnotetext{
${ }^{1}$ Metaphors of madness become "swollen to violent spectacles" (Bellin 141)

${ }^{2}$ Et dont la traduction du titre original Angst, très littéralement " angoisse ", montre bien à quel point des contresens dommageables peuvent se produire dans l'industrie cinématographique.
} 
supplémentaire la dimension pathographique de leur entreprise narrative : Clean, Shaven (1994) du réalisateur new-yorkais Lodge Kerrigan et Spider de David Cronenberg (2002) concentrent tous leurs efforts d'écriture cinématographique à dire les maux de la condition schizophrénique comme un état de souffrance sans néanmoins renoncer à investir cet état pathologique par l'imaginaire d'un récit. Après quelques remarques concernant l'approche et le rapport respectifs que ces films entretiennent avec la schizophrénie, je me propose d'étudier plus spécifiquement, en m'appuyant sur deux brefs extraits, la manière dont, dans l'un comme l'autre, la parole du schizophrène lutte pour se faire entendre et devenir voix.

\section{Clean, Shaven}

Clean, Shaven is the disturbing portrayal of schizophrenic's tormented search for his daughter and their short-lived reunion. [...] It is an intense and sometimes violent film that is fractured and abrupt in style.

La notice officielle du film le résume en ces quelques mots et ne cache pas son jeu. Le premier longmétrage de Lodge Kerrigan est une plongée en apnée au cœur des affres de la condition du schizophrène.

Le film est court (une heure et quinze minutes), abrupt, aride, opaque et cryptique, comme l'ont souligné la plupart des commentateurs. Dérangeant aussi, comme dans la scène où Peter, sous la douche, se taillade le crâne à l'aide d'une paire de ciseaux, à la recherche d'implants qu'il imagine dissimulés sous sa peau. Cette violence n'est toutefois pas celle dont il a été question précédemment. Plutôt que d'offrir au public un spectacle de la violence dans lequel la fonction du fou, vecteur de peur et emblème d'altérité, est d'effrayer le spectateur tout en le confortant dans son sentiment de "normalité ", la violence de Clean, Shaven participe d'un effort pour saisir la dimension autodestructrice de la maladie mentale. Je ne fais ici que reprendre les propos du réalisateur, qui déclare avec une parcimonie caractéristique : 
The film is disturbing. Sometimes it is very violent. However, the violence comes solely out of an attempt to understand the brutal and destructive aspects of mental illness.

La gageure de Lodge Kerrigan dans Clean, Shaven est en effet de percer cette couche d'hermétisme qui caractérise la condition schizophrénique, d’accéder à ou du moins de nous rapprocher de leur incompréhensible réalité comme le rappelle Racamier quand il écrit que « l'énigmatisme est pour les schizophrènes comme une règle de vie ; qui les comprend ne comprend rien, mais qui ne comprend plus commence à les comprendre »(47). Pendant les 95 minutes du film, nous sommes ainsi embarqués aux côtés de Peter Winter qui cherche à retrouver sa fille et semble à chaque instant en lutte avec lui-même pour ne pas s'effondrer, hormis peut-être dans les quelques minutes durant lesquelles, à la fin du film, il parvient à ré-investir son statut de père. Difficile d'imaginer un film dans lequel la proximité spectatorielle avec le personnage principal pourrait être plus grande, quand bien même il ne fait nul doute que sa folie est d'un ordre radicalement " hétérologique ", inaccessible à nos capacités de décodage rationnel ou aux schémas cognitifs auxquels l'espace du cadre cinématographique et le film de fiction nous ont habitués. L'identification, même secondaire, est entravée, et aucun reflet n'est possible. Sans doute est-ce l'une des façons de lire ces plans répétés dans lesquels Peter cherche désespérément à abolir son propre reflet en retournant tous les miroirs et en opacifant compulsivement les vitres et les rétroviseurs de son véhicule.

Le travail préparatoire du réalisateur fut intense. Kerrigan a passé trois ans à effectuer des recherches pour son film, a fréquenté des institutions pour psychotiques en tant que stagiaire pendant près d'un an. Le projet initial du film semble également avoir été catalysé par le fait d'avoir vu un de ses plus proches amis sombrer dans la schizophrénie à l'âge de dix-sept ans. De toute évidence, Clean, Shaven n'est pas un film qui cherche à manipuler ou à instrumentaliser la schizophrénie à des fins cinématographiques conventionnelles. Il s'agit au contraire d'une entreprise filmique qui met le cinéma au service de l'interrogation sur la nature de la schizophrénie comme pathologie, dans une démarche proprement pathographique. A ce stade, il est presque remarquable 
de constater que Clean, Shaven, quoique minimale, est quand même une fiction, convoquant de ce fait l'imaginaire dans un processus d'écriture cinématographique et de narrativisation de la maladie. Cette fictionnalisation s'est vue validée comme "dramatic rightness ${ }^{3}$ » -- droiture ou rigueur dramatique, un terme qu'il convient peut-être d'entendre de façons spatiale et morale dans un film décrit ailleurs par le même critique comme un parcours de la douleur en zigzag, « agonized schizophrenic zigzag ». Une manière peut-être de tendre un fil, aussi fragile soit-il, entre l'expérience du spectateur et celle du psychotique, décrit par Patrick Fuery comme le spectateur cinématographique par excellence ${ }^{4}$.

La métaphore du fil peut se tisser en arantèle quand on se tourne vers Spider de David Cronenberg, adapté du roman de Patrick McGrath, sorti sur les écrans français en 2002, autre voyage cinématographique dans l'espace turbulent de la schizophrénie. Le personnage éponyme, interprété par Ralph Fiennes, est embarqué dans une anamnèse douloureuse. Dès la séquence inaugurale du film qui nous montre l'arrivée d'un train en gare de Londres, il nous apparaît d'emblée comme barré, inaccessible. Les vingt premières minutes du film nous font suivre un individu entièrement opaque, aussi muré que les façades aveugles des maisons de l'East End londonien devant lesquelles il passe. Pendant cette première partie du film, assez longue, c'est le parti-pris de l'hermétisme que Cronenberg semble retenir. Le personnage, claustré dans sa maladie, mutique, ressemble très fortement à Peter Winter de Clean, Shaven, ainsi qu'à un autre schizophrène, bien réel celui-ci, filmé par Raymond Depardon dans son documentaire San Clemente, un homme grand et ténébreux, enveloppé dans son manteau, parfaitement hermétique au regard " transparent » de la caméra. Le sentiment d'opacité est presque aussi fort pour le spectateur du film de Cronenberg qu'il l'est dans

\footnotetext{
${ }^{3}$ [T] here is a dramatic rightness to it. And partly because the film is so unsettling, partly because it so nakedly and persuasively makes an end run around movie-studio conventions, it seems neither voyeuristic nor exploitative. What makes this film terrifying and profoundly sad and moving is its empathy with its protagonist's torment. (Boston Globe Review, May 26, 1995)

${ }^{4}$ the cinematic spectator may not be psychotic, and cinema does not actually bring on psychotic episodes, but in many ways the psychotic is the cinematic spectator par excellence. (Fuery 102)
} 
celui du documentariste, à la différence près que dans le cas de Spider, comme par une sorte d'impératif fictionnel catégorique, l'imaginaire va finir par intervenir sur cette réalité en crise. Et c'est justement par l'entremise de productions d'images paradoxales, à travers un dispositif complexe mêlant regards, analepses, écriture, focalisation et parole que s'effectue in fine l'impossible pari de la traversée du miroir qui va permettre d'embarquer le spectateur dans l'infini turbulent qui anime le personnage de Spider pour enfin accéder à son intériorité erratique.

Il est intéressant de noter que dans le roman de McGrath, inspiré des lectures de John Laing sur le faux-moi (The Divided Self, 1961), le récit homodiégétique du personnage participe simultanément, par des mécanismes de dédoublement et de fluctuations entre niveaux d'écriture et de voix, à sa construction/ déconstruction. Spider explore ainsi la division fonctionnelle entre la vie mentale du «Moi-observant et celle du Moi- éprouvant qui rend compte de certains « délires d'observation » des commentaires hallucinatoires des actes et de la pensée " du schizophrène (Bourgeois, 82), rendant ainsi possible le retour vers le passé et la quête de l'événement traumatique de son histoire derrière le double écran de la réalité (Spider a tué sa mère) et de la délusion (II a tué sa mère parce qu'il croyait qu'elle n'était pas sa mère, mais une usurpatrice). C'est dans cette faille que s'immisce le "Moi-racontant », l'espace imaginaire du film et son récit malade, dont on aurait pu penser dans un premier temps qu'il aurait privilégié le recours à la voix-off, tout d'abord envisagé par le romancier qui a également signé le scénario du film. II n'en fut finalement rien. Dans Spider, c'est bien en faisant le pari de l'image, fût-elle filmique, hiéroglyphique, hallucinatoire ou « schizographique " que Cronenberg nous permet d'entrer dans l'intimité du sujet malade, et d'explorer les plus sombres recoins « d'un être totalement renfermé dans son monde intérieur, pratiquement incapable de communiquer avec les autres - sauf par d'illisibles gribouillages sur un petit carnet et par des grommellements presque inaudibles » (Grunberg 171).

Les « grommellements » du protagoniste de Spider et la démarche schizographique du réalisateur me permettent de m'intéresser à présent à quelques processus de verbalisation et de mise en scène de 
la parole du psychotique dans les deux films ici à l'étude. Constatons d'emblée que Clean, Shaven et Spider sont deux films dans lesquels la parole verbale n'est pas l'élément central de l'accès à l'imaginaire pathologique du personnage. Contrairement à ce qui se passe dans Keane (2004), autre film de Lodge Kerrigan, ou dans Frownland de Ronald Brownstein (2007), la parole n'est ni délirante, ni proprement psychotique. Au contraire, elle semble plutôt, dans un film comme dans l'autre, un élément problématique dans le rapport du sujet à autrui et à la réalité qui l'entoure, presque une impasse. Ceci n'est évidemment guère surprenant si l'on considère que les deux films explorent sur un mode cinématographique la question de la psychose, qui est avant tout une perte de contact avec la réalité et donc avec autrui, une expérience pouvant confiner à l'effondrement $a$-symbolique, ou s'abolissent langage et parole dans un processus d' « inanisation » du Moi.

Spider et Clean, Shaven sont donc deux films dans lesquels la parole est présentée comme algique, l'expérience douloureuse d'une intersubjectivité mise en péril par la maladie. Les mots, dans un premier temps, ne sortent pas. II faut attendre presque vingt minutes dans chacun des films pour qu'interviennent les premières paroles prononcées par les protagonistes. Dans Clean, Shaven, il s'agit d'un face-à-face entre Peter et sa mère, une scène au cours de laquelle il est patent que la communication échoue à s'établir, que l'échange est impossible. La première réplique de Peter est une question à laquelle sa mère répond symptomatiquement par: "it's broken », comme pour mieux lui (et nous) signifier la rupture du lien. Quand à son tour, il lui répond pour la première fois, et par la négative, il demeure hors-champ. La scène, malgré une construction presque conventionnelle de champ-contrechamp, prend alors la tournure d'un dialogue en échec, la mère procédant par injonction 'Eat'/ 'Listen to me' / 'why don't you go to your room', tandis que la question de Peter Where's Nicole ? - demeure sans réponse. Le très gros plan sur les lèvres maternelles, à deux reprises, souligne, par un processus de subjectivation, plus complexe qu'il n'y paraît, la terrible hétérogénéité de la parole de l'autre, même quand cet autre est la mère. 
Cette scène peut être mise en miroir avec une séquence de Spider, à la différence notoire que nous plongeons à cet instant dans un niveau de réalité différent, celui des souvenirs du personnage, alors qu'il re-convoque à sa mémoire, par l'entremise de son carnet et d'un processus d'inscription solitaire, une conversation avec sa mère dans la cuisine de son enfance.

II y aurait sans doute beaucoup à dire sur les questions de la mère et de la filiation dans ces deux films, ainsi que dans la schizophrénie en général, mais de telles considérations n'entrent pas dans mon propos d'aujourd'hui. Concentrons-nous ainsi sur la mise en scène de la parole et les modalités de son fonctionnement. Il faut aussi préciser que lorsqu'intervient cette séquence, comme dans celle de Clean, Shaven, le spectateur n'a pas encore entendu parler le protagoniste, enfermé dans sa pathologie, en situation d'effroi mutique par rapport à un réel qui ne cesse de l'assaillir. Cette claustration est d'ailleurs encore soulignée par les premiers plans de la séquence, dans lesquels Spider se tient seul dans sa mansarde et griffonne dans son journal. Lorsque l'analepse se déclenche dans le plan suivant, introduite par un cut plutôt qu'un fondu enchaîné qui en aurait trop dit, l'ambiguïté demeure encore quelques instants: où sommes-nous exactement ? Qui est derrière ce rideau soulevé ?

Intervient alors la parole. D'abord celle de la mère, suivie de celle de l'enfant, reprise en écho par l'adulte derrière le carreau, le temps de deux répliques, avant que ne se produise l'inversion écholalique qui bouleverse l’organisation du récit : Spider adulte énonce alors en premier les phrases qui seront ensuite répétées par l'enfant. C'est à cet instant précis, par un subtil déplacement du locus de l'énonciation que s'effectue une réattribution de la parole et une reconfiguration du fonctionnement du récit: Spider adulte devient à cet instant le narrateur de son enfance. Le film peut alors devenir, comme il le fera ensuite de manière plus systématique, le récit filmique (qui inclut donc aussi la part verbale du texte cinématographique) d'une quête douloureuse vers le passé, une quête impossible visant à recoller les morceaux d'une histoire familiale brisée et ensanglantée, à l'image du carreau brisé de l'asile auquel Spider amènera le fragment manquant, sans néanmoins 
pouvoir espérer le réparer totalement. Demeure la ligne de faille, la coupure avec le réel, la vie hors de soi, " déporté » (Racamier 201), qui fait que le schizophrène échouera finalement à achever son récit autrement que par sa propre annihilation (suicide dans le roman, retour à l'asile dans le film).

In fine, Il demeure désespérément pris dans un processus d'écriture voué à l'échec car, pour le dire avec Paul-Claude Racamier, « écrire intérieurement sa vie, chacun le fait sans cesse, le névrosé le fait en hiéroglyphes, et le psychotique sur un écran qui ne prend pas l'encre. » (192)

Jocelyn Dupont

VECT-Mare Nostrum (EA 2983)

\section{Bibliographie}

Bellin, Josuah David. Framing Monsters: Fantasy Film and Social Alienation. SIU Press, 2005.

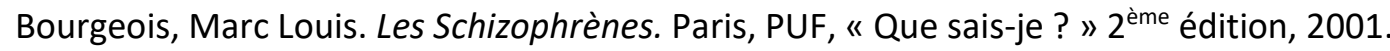

Fuery, Patrick . Madness and Cinema. Psychoanalysis, Spectatorship and Culture. Palgrave MacMillan, 2004.

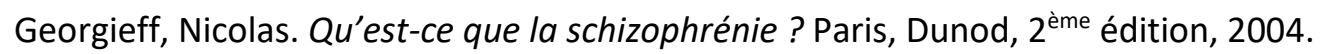

Racamier, Pierre-Claude. Les Schizophrènes, 1980. Petite bibliothèque Payot, 2001. 